Opinion

\title{
Establishing and Evaluating a Multidisciplinary Community-Based Sleep Clinic for Children with Neurodevelopmental Difficulties in Inner London
}

\author{
Jessica R Turnbull *, Sally Hobson, Jenna Vyas-Lee, Bidisha Lahoti
}

Guy's and St Thomas' NHS Foundation Trust Community Children's Services, Sunshine House Children and Young People's Development Centre, 27 Peckham Road, London, SE5 8UH, UK; E-Mails: jessica.turnbull@gstt.nhs.uk; $\quad$ sally.hobson@gstt.nhs.uk; $\quad$ jenna.vyas-lee@gstt.nhs.uk; Bidisha.Lahoti@gstt.nhs.uk

* Correspondence: Jessica Turnbull; E-Mail: jessica.turnbull@gstt.nhs.uk

Academic Editor: Bart Ellenbroek

OBM Neurobiology

2021, volume 5 , issue 2

doi:10.21926/obm.neurobiol.2102101
Received: January 30, 2021

Accepted: June 01, 2021

Published: June 11, 2021

\begin{abstract}
We describe our experience of setting up and evaluating a community-based multidisciplinary sleep service for children with neurodevelopmental disorders and psychosocial vulnerabilities. Referrals are accepted for children (1-18 years of age) with persistent sleep problems, and neurodevelopmental difficulties or significant psychosocial adversity, living in two inner-city boroughs. Holistic sleep assessment involves 1-hour Paediatrician-led consultation, often followed by Psychologist-led parent workshop, with follow-up telephone/face-to-face contacts to support implementation of behavioural interventions. Acceptability is measured through parent feedback; efficacy measured through Composite Sleep Disturbance Index, Sleep Problem rating, and locally developed Burden Score. Referrals for 354 children were received in the first two years. 99\% of families report the service met their child's needs well/very well. Sleep studies were accessed for around a quarter of children. Medical and social complexity was common, and more prevalent in families who were less able to engage to completion of intervention (significant psychosocial vulnerability in $43.4 \%$ of suboptimally engaged, versus $22.7 \%$ of engaged group). Clinical outcome measure scores improved for a subset of families in whom data was available, who had been able to engage in the intervention. There has been a high demand for our community-based multi-
\end{abstract}

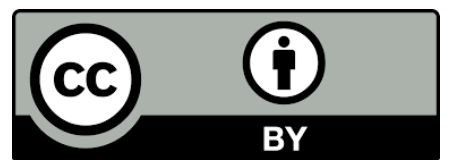


disciplinary sleep service, with positive feedback from families. Intervention has resulted in positive impacts on sleep outcome measures for those engaged. There continue to be barriers to accessing intervention, and putting in place recommendations, for some families which requires further evaluation and potential service development. We believe the communitybased multi-disciplinary model of this service could be readily replicated in other localities to benefit sleep health on a wider scale.

\section{Keywords}

Sleep; neurodisability; neurodevelopment; children

\section{Background}

Sleep-related difficulties amongst children with neurodevelopmental disorders are extremely common, affecting $60-80 \%$ of children with autism spectrum disorder (ASD), attention deficit/hyperactivity disorder (ADHD), and other neurodevelopmental conditions [1-3]. Sleeprelated difficulties encountered in this group include difficulties with sleep initiation and maintenance, circadian rhythm disturbances, and primary sleep disorders such as sleep-disordered breathing and restless legs syndrome/periodic limb movements in sleep [4]. Co-occurring mental health, neurodevelopmental, behavioural, and medical conditions can further contribute to sleep disturbance, and can shape the recommendations and strategies needed to address the reported sleep disturbance [4]. Sleep deprivation in children negatively impacts mental health, behaviour, learning, immunity, healthy weight maintenance, growth, parental mental health and family functioning, and is linked to increased use of physical chastisement [5-8].

Children presenting to Community Paediatricians in the United Kingdom include those at risk of a range of neurodevelopmental disorders (e.g. ASD, ADHD, Intellectual Disabilities of various aetiologies), physical and sensory disabilities, and social vulnerabilities. Sleep enquiry is a vital part of a holistic developmental assessment for this vulnerable population but is often not asked about [9], and therefore opportunities for intervention may be missed.

Two projects evaluating prevalence and impact of sleep-related difficulties in children with neurodevelopmental disorders living in Lambeth and Southwark were undertaken in 2016, including an informal survey of families attending the local Child Development Centre in Lambeth [personal communication] and a qualitative study of Primary and Secondary special-needs school populations in Southwark [10]. This project found that over $60 \%$ of participant children had reported difficulties with sleep. Around two thirds of this cohort had Autistic Spectrum Disorder (ASD) and we diagnose over 150 new children with ASD in each borough every year.

We approached our local commissioners with a business case to pilot a multidisciplinary, community-based sleep service for children with neurodisability for 2 years. We argued that providing local care for local children made clinical and economic sense. We had full support of our local tertiary children's sleep medicine service in the development and implementation of the service, which is costed to include paediatrician, psychologist, and administrative support time, with the aim of identifying and diagnosing sleep-related problems as early as possible, advising parents, 
prescribing medications where relevant (with shared care arrangements with General Practitioners (GPs)), and running parent training workshops and professional teaching.

The service was designed at the outset to self-evaluate to monitor acceptability and efficacy by recording both parent/client satisfaction and validated clinical outcome measures, and has evolved to reflect areas where we felt the initial model could be improved.

We report our evaluation of the service over the first two years of running, which reflects a clinical service rather than a research project, and discuss the utility of this model in the context of our deprived inner-city populations. We believe the service model could be easily replicated and adapted to meet the differing needs of populations in other localities.

\section{Service Set-Up, Running, and Demographics}

The service was designed to accept referrals for children (1 - 18 years) with GPs in either of the two local boroughs served by Evelina London Community Children's Services (Lambeth and Southwark), with a neurodevelopmental or significant psychosocial vulnerability and persistent sleep difficulty.

A preliminary analysis (Figure 1) was undertaken in August 2018 of the 223 referrals received at that stage (Table 1).

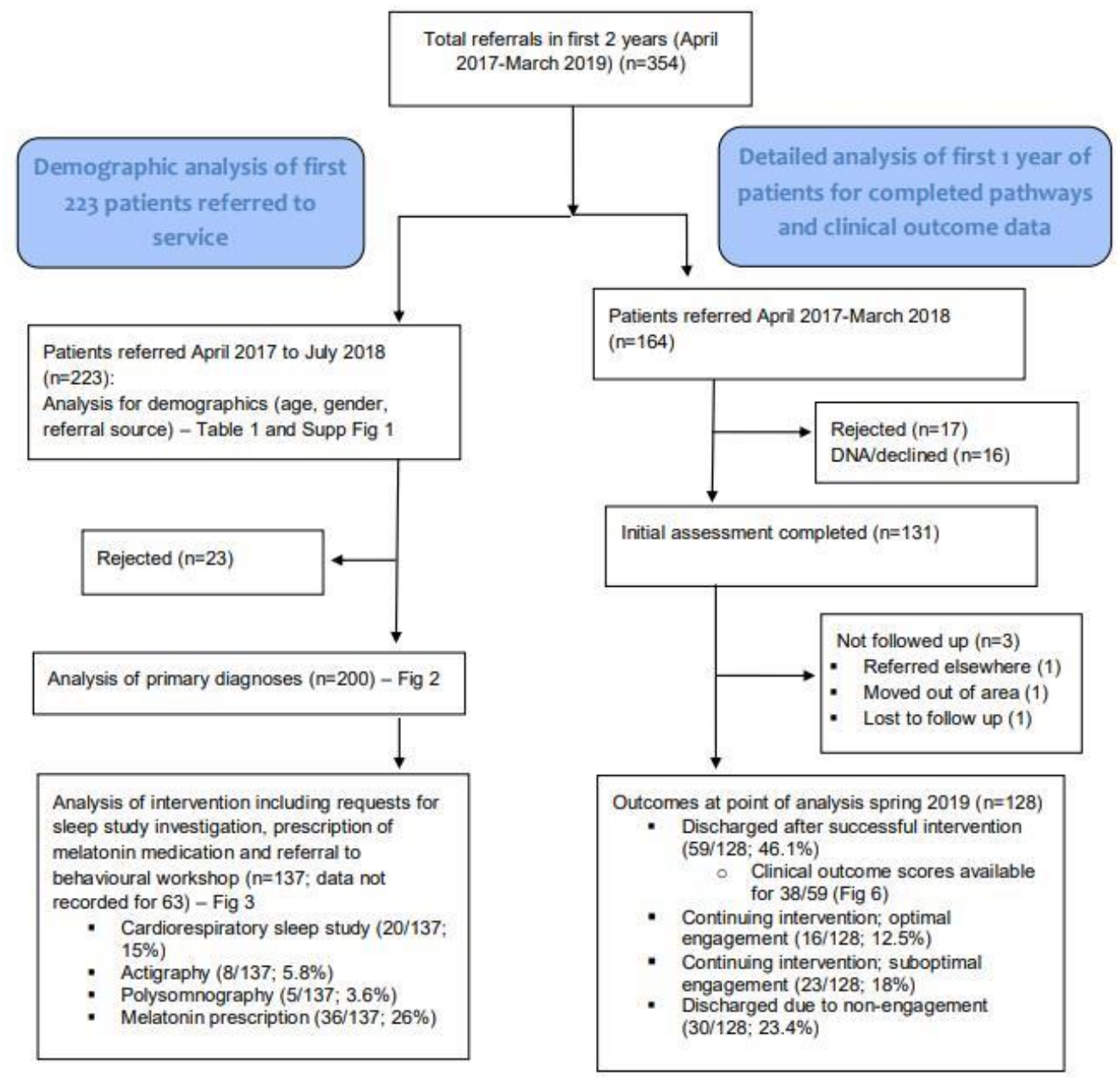

Figure 1 Flow diagram of process of service evaluation. 
Table 1 Demographics of children referred April 2017 to July $2018(n=223)$.

\begin{tabular}{ll}
\hline Gender: & \\
\hline Male: & $152(68 \%)$ \\
Female: & $71(32 \%)$ \\
Referral source: & \\
Community Paediatrician: & $169(76 \%)$ \\
School Nurse or Therapist: & $15(7 \%)$ \\
GP: & $14(6 \%)$ \\
CAMHS: & $13(6 \%)$ \\
Education professional: & $7(3 \%)$ \\
Hospital Paediatrician: & $5(2 \%)$ \\
\hline
\end{tabular}

Twenty-three referrals were rejected; reasons for rejection included child not meeting referral criteria of having neurodevelopmental/psychosocial difficulties, and sleep management best undertaken by a different team (e.g. children already working closely with Child and Adolescent Mental Health Services (CAMHS) on behaviour and sleep).

Age of children referred broadly reflects the ages of children usually referred to Community Paediatric clinics, with most in the 2-10 year range (Supplementary Figure 1).

Neurodevelopmental profile of children referred includes a high prevalence of autistic spectrum condition (in $>50 \%$ ) and diagnostic complexity ( $25 \%$ having 3 or more significant problems recorded; Figure 2).
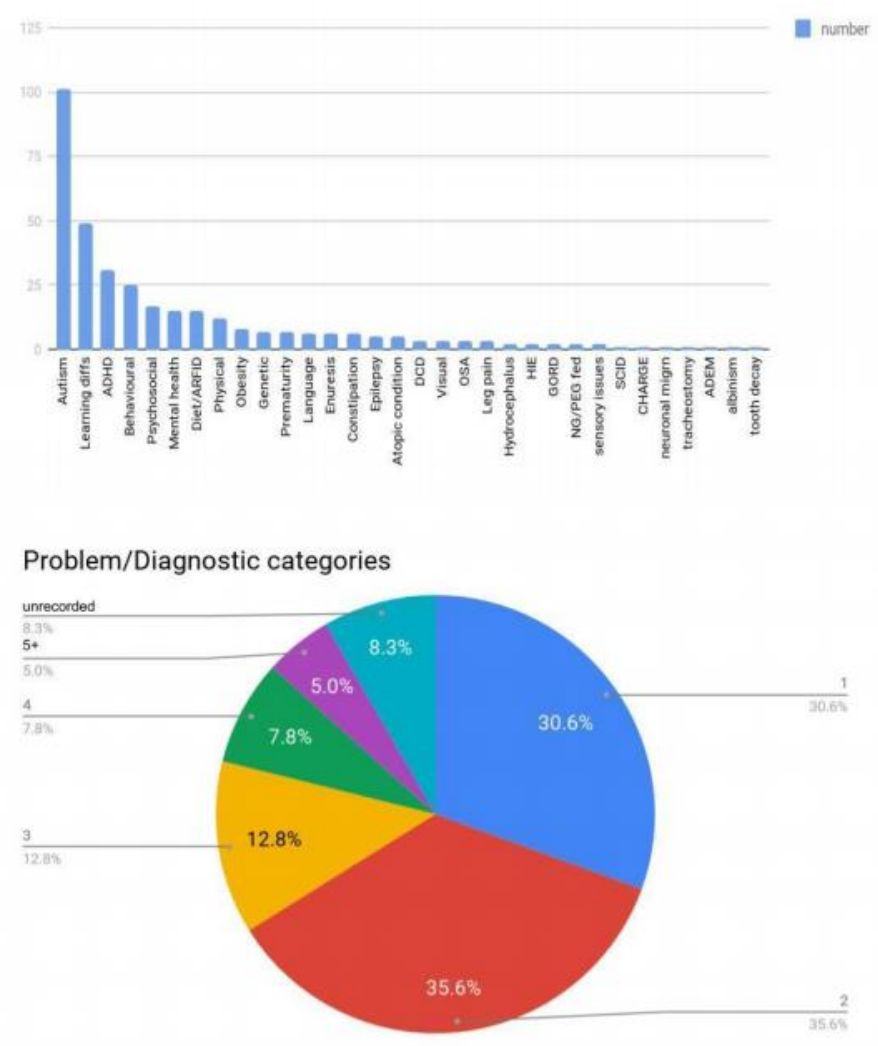

Figure 2 Problems and diagnoses recorded for children at clinical assessment $(N=200)$. 
The current clinical team consists of: Consultant Paediatrician 0.2 WTE (whole-time equivalent), Specialty Doctor 0.3 WTE (combined in total delivering 10 doctor-led clinics/month), Band 8 Clinical Psychologist 0.4 WTE, Band 5 Assistant Psychologist 0.5 WTE and Band 4 Administrator 0.5 WTE. The current clinical composition reflects a slight expansion of the service following the pilot two years, to meet demand, with an increase in doctor-led clinics from 8 per month to 10 per month (with the aim of maintaining wait-times at no more than 3 months), and an increase in Band 8 Psychologist time from 0.2 WTE to 0.4 WTE to provide intervention work to the large number of families we determined required this part of the service.

\subsection{Initial Assessment}

A one-hour doctor-led (Consultant or Specialty Doctor) assessment aims to unpick the complex interacting factors which often contribute to a child's sleep difficulties. A proforma guides the clinician through a holistic assessment of the child's presentation, which in outline includes;

- Developmental profile, communication skills, mental and emotional wellbeing

- Medical problems (including asthma, eczema, constipation, obesity, restricted diet) that can directly affect sleep

- Family context - including parents' and siblings' health, development and sleep patterns

- Bedroom environment - place of sleeping, bedroom/bed sharing, electronic devices, noise and light levels

- Housing - crowding, safety, safeguarding issues including routine enquiry for domestic violence

- Bedtime routines/practices - including differences between weekday/weekend

- A detailed description of the sleep disturbance and response to it - aiming to identify sleep disorders (such as respiratory, parasomnias, circadian rhythm disturbances, restless leg symptoms and potential neurological episodes), and where parental responses may be modifiable in order to effect change.

- Specific enquiry about daytime sleepiness, napping, sleep attacks and symptoms associated with narcolepsy (cataplexy, sleep paralysis and hypnagogic hallucinations)

- Behavioural factors causing, maintaining, and exacerbating sleep issues (using an A-B-C approach to identify antecedents and consequences of behaviour)

- Parental beliefs, expectations, goals and what they have already tried

- Child/young person's views on their sleep, and their goals for intervention, willingness to engage and motivating factors

- Height and weight measurement where not previously available

- Physical examination as indicated

A formulation of the child's sleep difficulties taking aetiological, maintaining and exacerbating factors into account can then be discussed in the context of parental beliefs, goals and expectations. Management plans, the need (or not) for further investigation (sleep studies or blood tests), medication (usually melatonin), behavioural intervention, or a combination will be agreed, alongside brief psychoeducation regarding sleep physiology and advice about behavioural interventions. Where needed, the child may be referred to specialist services, for example to Paediatric Neurology if red-flags for possible neurological episodes at night emerge, or to Gastroenterology for management of problematic gastrointestinal symptoms. 
For a more detailed description of how to assess and manage children's sleep difficulties see Reynolds and Malow [4], Turnbull \& Farquhar [11] and McDonald \& Joseph [1].

\subsection{Medical Intervention}

Following initial assessment, some children are referred for sleep studies (Figure 3), where concerns exist in relation to sleep-disordered breathing, unusual episodes during sleep, or more detailed objective measures of sleep are required. These are undertaken at the local tertiary children's sleep medicine unit. Of a subset recorded $(N=137)$, this included 20 (15\%) referred for cardiorespiratory sleep study, 8 (5.8\%) for actigraphy, and 5 (3.6\%) for polysomnography.

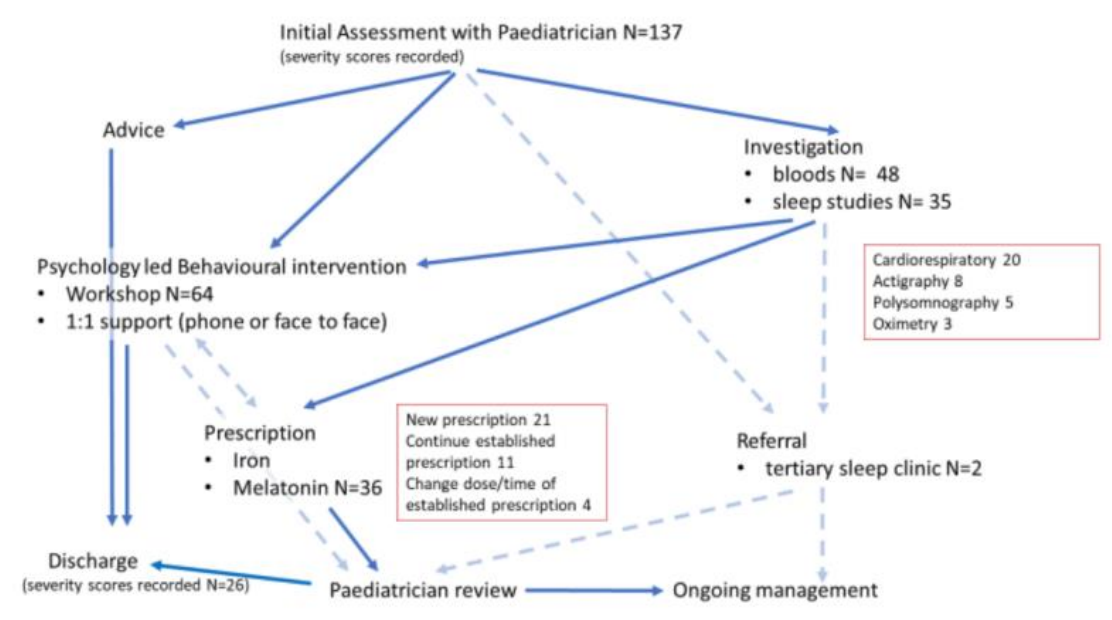

Figure 3 Pathway through the service.

Some children are recommended to have blood investigations, where concerns about Restless Legs Syndrome or Periodic Limb Movements in Sleep exist, with the aim of identifying and correcting iron deficiency (specifically low ferritin levels) [12-14]. This has been particularly relevant within the group of children with restrictive feeding practices in the context of autism spectrum disorder [15]. Of 21 sets of blood results reviewed, 8 children had ferritin levels in the deficient range $(<22 \mathrm{mcg} / \mathrm{L})$, and all 21 children tested had ferritin levels $<50 \mathrm{mcg} / \mathrm{L}$, the level recommended to treat in the context of concerns for possible Restless Legs Syndrome [12]. A trial of iron supplementation has been recommended where appropriate (very low ferritin levels $(<22 \mathrm{mcg} / \mathrm{L})$, or moderately low ferritin levels $(22-50 \mathrm{mcg} / \mathrm{L})$ with accompanying symptoms suggestive of Restless Legs Syndrome that appear to be impacting sleep).

Approximately a quarter of children we have seen were either already receiving, or were prescribed melatonin medication as part of their package of intervention. Melatonin medication has been shown to be helpful for improving sleep patterns in children with autism spectrum disorders [16]. Our discussion with families includes a strong emphasis on the use of melatonin alongside implementation of behavioural change, and that without concurrent optimisation of sleep practices, melatonin use alone may be unsuccessful. We encourage use of melatonin medication to help set new sleep practices in place, with the aim of withdrawing once improved sleep patterns are established. Our practice has been to initiate melatonin medication at the lowest efficacious dose, usually starting at $1-2 \mathrm{mg}$ at an agreed set time in the evening, increasing if needed in weekly intervals, to a maximum of $6 \mathrm{mg}$. If not effective at this dose, our practice is to recommend 
discontinuing rather than increasing further. Prescription of melatonin medication is under a shared-care agreement with local GPs, with secondary care (our clinic) providing the initial prescription and follow-up, and on-going prescription via the GP once established, and with guidance for a break every 6-12 months to establish whether melatonin medication is still required, and to maintain efficacy by reducing background levels.

Sleep diagnoses within our cohort have included behavioural insomnia of childhood (very commonly sleep-onset association type, but also limit-setting type), bi-phasic sleep patterns, delayed sleep-wake phase, and occasionally sleep-disordered breathing.

Only 2 patients assessed in the first 2 years were felt to require referral to the local tertiary service for further assessment, advice, or a second opinion.

\subsection{Psychology Intervention}

Around half of the families we have seen have been directed into a psychologist-led intervention following initial assessment, mostly accessed through a 3-hour parent workshop. Workshops are offered every 8 weeks, with the location alternating between boroughs to maximise accessibility and acceptability. During the first two years of the service, the maximum wait-time for a workshop was 8 weeks. Each workshop was attended by a maximum of 10 sets of parents. The workshop covers: psychoeducation about sleep physiology, control of sleep, how sleep problems arise, and strategies to intervene; and moves on to consider specific situations and form individualised plans for families.

Feedback from those who attended workshops was consistently positive. From a random sample of 15 feedback forms, 14 rated the workshop "very good"; 1 rated "good". All 15 forms stated attendees would recommend the workshop to other parents, and that the workshop met their needs.

Key take-home messages recorded on feedback forms included: use of sleep hygiene strategies such as reducing screen time, increasing physical activity levels in the daytime, and having a consistent bedtime. Suggested improvements for future workshops included: more unstructured time for parents to discuss issues together, and more information related to specific issues such as separation anxiety.

Following the workshop, weekly support is offered by telephone. These sessions follow a manualised approach which could be adapted to suit the needs of the family, with a different focus each week: consolidating psychoeducation, formulating current difficulties including working on factors perpetuating sleep issues, motivational interviewing, troubleshooting, and acknowledging and validating psychosocial stressors as barriers to progress.

Occasionally one-to-one, face-to-face intervention with the parent is preferable (e.g., an interpreter is required, or parents' learning or social vulnerabilities preclude attending a workshop), or to facilitate direct work with adolescents. The decision to offer one-to-one intervention is made jointly as a multidisciplinary team following initial assessment, or following the workshop. Approximately $25 \%$ of families have required at least one individual session. These sessions take place in the clinic setting; interpreters attend alongside families where necessary, and after-school appointments are prioritised for young people. Where needed, the initial one-to-one session focusses on the same content as the group workshops, with adaptations to suit the needs of the family. 


\section{Clinical Service Evaluation}

\subsection{Acceptability}

Feedback forms are given at clinic assessment asking, "How well did the appointment meet your / your child's needs?", and "How likely are you to recommend the service?" with free-text additional comments.

Families rated the clinic assessment positively; $80 \%$ self-reporting as "very likely" to recommend the service to others ( $19 \%$ "likely"), and $86 \%$ reporting their child's needs were met "very well" (13\% "well").

\subsection{Efficacy}

Outcome measures are scored at initial assessment and at completion of intervention, including Composite Sleep Disturbance Index (CSDI; 12-point severity rating across 6 sleep-related domains, with 5-point sleep dissatisfaction rating - higher scores indicating more problems), a Problem severity rating (large/moderate, or small/no problem) from the work of Hiscock [17] and a locallydeveloped Burden Score, rating perceived impact on carer/wider family and degree of worry/anxiety about the child's sleep (scored 0-6; higher score indicating greater impact; Table 2). The CSDI has been used as a validated tool for measuring sleep disturbance in children in previous studies $[18,19]$.

Table 2 Burden score.

\begin{tabular}{lll}
\hline Question & Response & Allocated score \\
\hline \multirow{2}{*}{$\begin{array}{l}\text { How much do your child's sleep difficulties } \\
\text { impact on you as a parent? }\end{array}$} & Great impact & 2 \\
& Somewhat of an impact & 1 \\
& No impact & 0 \\
How much do your child's sleep difficulties & Great impact & 2 \\
impact on the family as a whole? & Somewhat of an impact & 1 \\
& No impact & 0 \\
Are you ever worried or anxious about your & Often & 2 \\
child's sleep? & Sometimes & Never \\
\end{tabular}

Detailed analysis of the first 164 children referred (April 2017 to end March 2018) was undertaken for patient pathways and completed clinical outcome data (Figure 1). Due to time and resource constraints the availability and timing of post-intervention outcome recording is variable.

Clinic Paediatricians (SH and JT) analysed patient records, categorising patients in terms of their "engagement" with the service, and "success" in terms of parent-reported change in sleep, conferring as necessary to resolve any unclear situations.

Patient pathway end-points:

- Engagement with the service: 
- "Optimal engagement" = able to access and engage with the service and interventions offered

- "Sub-optimal engagement" = partial engagement (e.g. inconsistent response to prearranged contacts, inconsistent implementation of strategies), or non-engagement leading to discharge from service before intervention complete

- Degree of success:

- Parent/young person report of complete/partial or no improvement

- Clinical outcome scores (repeat CSDI, Problem and Burden scores)

At the point of analysis $46 \%$ (59 out of 128 families seen and followed-up) had engaged with intervention to successful discharge. A further 30\% (39 out of 128) were receiving on-going support/intervention. Decision to discharge was mutually agreed between clinician and family, and could be at the point that:

- sleep had improved to an acceptable level for the family

- the family had confidence to continue to implement strategies without the need for ongoing support

- clinician and family agreed there were persisting barriers to progress, or competing priorities, meaning that it was agreed further improvement in sleep patterns was unlikely to be achieved at that stage

Although we developed structured intervention pathways (initial design of assessment +/psychology-led workshop followed by up to 6 telephone contacts with psychologist), we found the needs of children and families seen in the clinic varied greatly, with the minimum number of contacts required being one (children seen once and discharged with advice), up to a maximum of 24 contacts (family experiencing significant psychosocial adversities and inconsistent engagement). Actions included face to face contacts, attempted agreed phone contacts, and successful phone contacts. The mean and median number of actions was 8 (Figure 4).

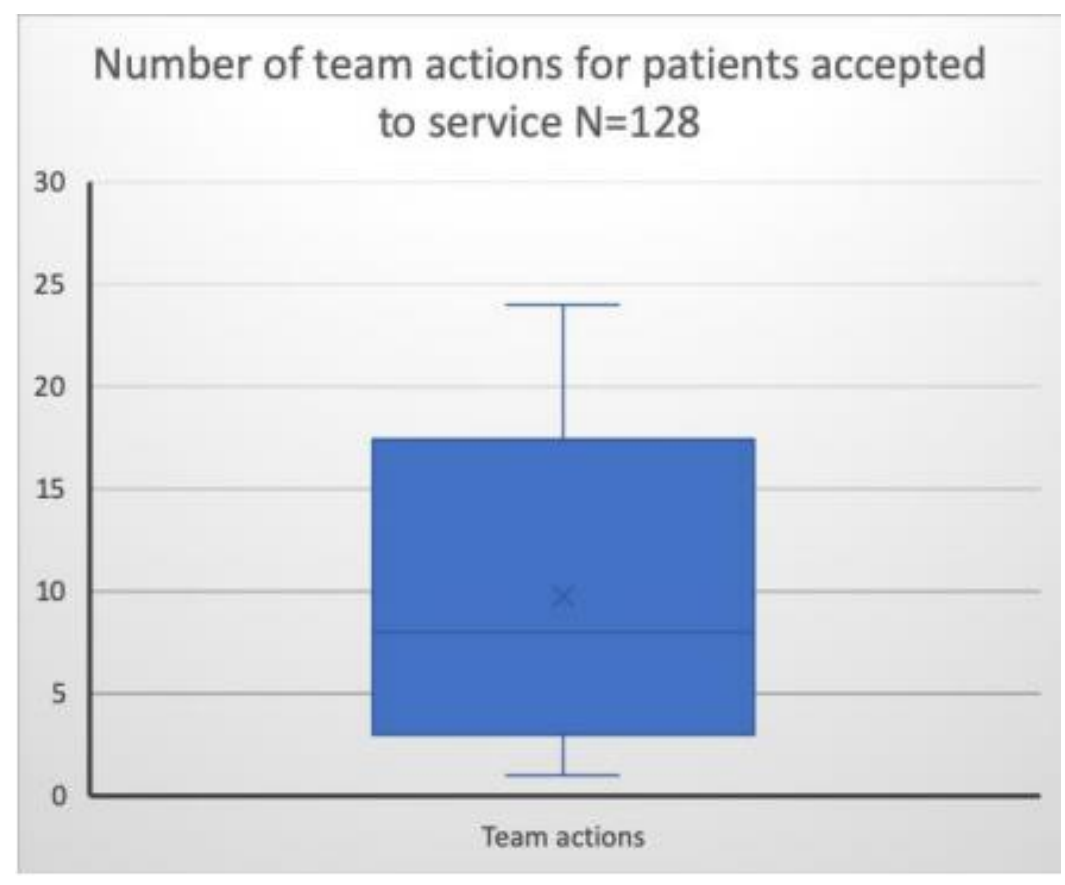

Figure 4 Number of team "actions" for patients ("action" defined as face to face contacts and attempted or successful agreed telephone contacts). 
We reviewed records for complexities as follows:

- Safeguarding concerns (Child Protection Plan, Child in Need Plan, or Team Around Child/Family in place)

- Significant housing factors (housing issues recorded in correspondence as significantly impacting children's sleep and interventions e.g. children sleeping in the lounge with siblings due to overcrowding)

- Complex medical problems (e.g. child on overnight PEG feeding, complex epilepsy, complex motor disorders)

Analysis found higher rates of complexity in the families who were less able to engage $(43.4 \%$ of sub-optimally engaged group, versus $22.7 \%$ of those fully engaged; (Figure 5 ).
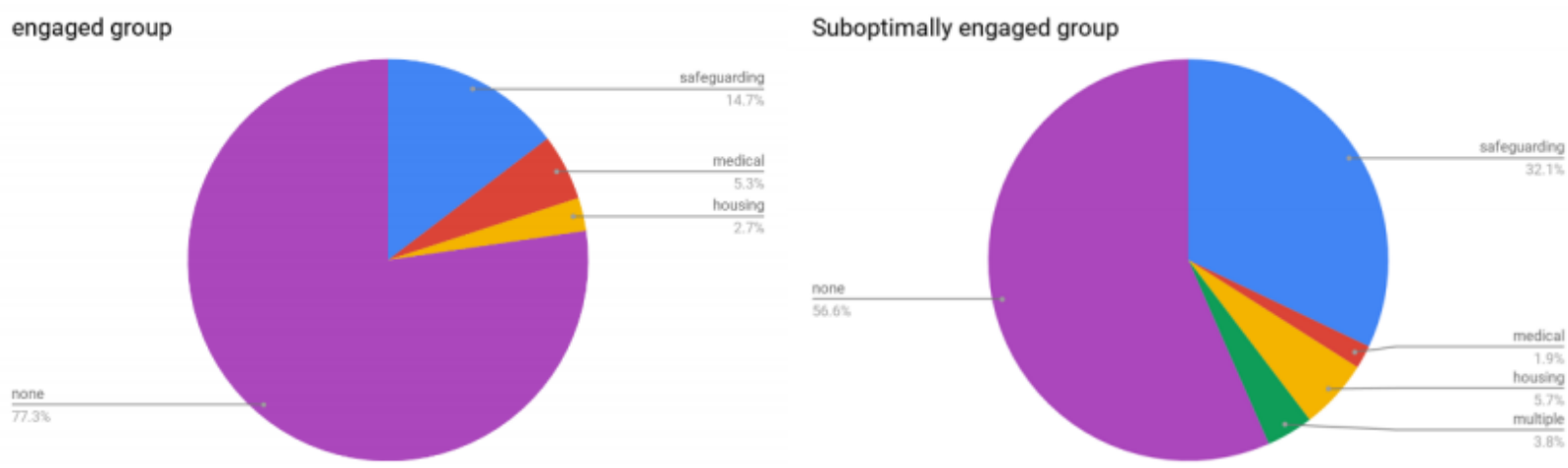

Figure 5 Identified vulnerabilities in engaged $(N=75)$ versus suboptimally engaged $(\mathrm{N}=53)$ groups.

\subsection{Clinical Outcome Score Evaluation}

We analysed clinical outcome measure scores, available for 38 children, representing $64.4 \%$ of the 59 who engaged to the point of discharge.

We categorised the 38 children into two groups:

1. Discharged with advice after initial assessment, contacted later for repeat outcome measures $(\mathrm{N}=6)$

2. Referred for, and engaged with, psychology intervention to point of discharge $(N=32)$

Analysis demonstrated overall improvements in all outcome measures in this subgroup [Table 3 and Figure 6]. 


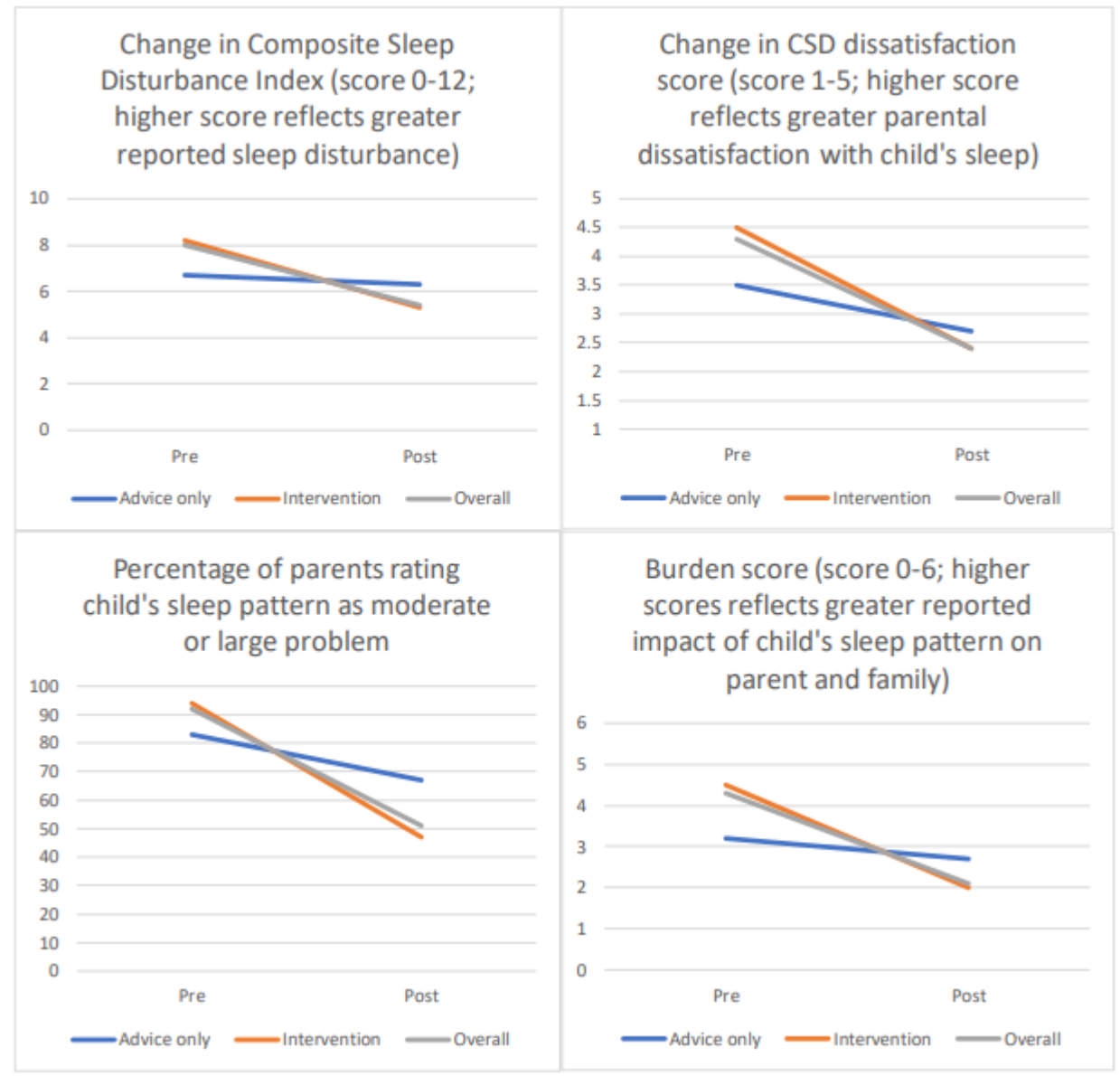

Figure 6 Change in clinical outcome measure scores for those engaged to point of discharge (Advice only $\mathrm{N}=6$; Intervention $\mathrm{N}=32$; Overall $\mathrm{N}=38$ ).

Table 3 Change in clinical outcome measure scores for those engaged to point of discharge $(\mathrm{N}=38)$.

\begin{tabular}{|c|c|c|c|c|c|c|c|c|c|}
\hline \multirow[t]{2}{*}{ Outcome Scores: } & \multicolumn{2}{|c|}{$\begin{array}{l}\text { CSD index } \\
(0-12)\end{array}$} & \multicolumn{2}{|c|}{$\begin{array}{l}\text { CSD } \\
\text { dissatisfaction } \\
(0-5)\end{array}$} & \multicolumn{2}{|c|}{$\begin{array}{l}\% \quad \text { rating } \\
\text { moderate } \\
\text { large problem }\end{array}$} & $\begin{array}{l}\text { as } \\
\text { or }\end{array}$ & \multicolumn{2}{|c|}{$\begin{array}{l}\text { Burden } \\
\text { score }(0-6)\end{array}$} \\
\hline & Pre & Post & Pre & Post & Pre & Post & & Pre & Post \\
\hline Overall & 8.0 & 5.4 & 4.3 & 2.4 & $92 \%$ & $51 \%$ & & 4.3 & 2.1 \\
\hline $\begin{array}{l}\text { Discharged with advice from } \\
\text { initial assessment, contacted } \\
\text { later for repeat data; } \mathrm{N}=6\end{array}$ & 6.7 & 6.3 & 3.5 & 2.7 & $83 \%$ & $67 \%$ & & 3.2 & 2.7 \\
\hline $\begin{array}{l}\text { Discharged following } \\
\text { psychology intervention; } \mathrm{N}=32\end{array}$ & 8.2 & 5.3 & 4.5 & 2.4 & $94 \%$ & $47 \%$ & & 4.5 & 2.0 \\
\hline
\end{tabular}

Clinical outcome measure change was greater for those who engaged in psychology intervention, than those who were given advice at clinic appointment only and subsequently discharged (e.g. 
Composite Sleep Disturbance Index improved from average 8.2 to 5.3 in full intervention group, versus 6.7 to 6.3 in advice-only group (max score 12 )). This may reflect that sleep problems identified at initial assessment were less severe and less burdensome in the group discharged with advice-only, with lower Dissatisfaction, Problem and Burden scores reported by parents at initial assessment in this group.

The group for whom clinical outcome score data was available was small compared to our overall cohort, and therefore this data can only be interpreted with caution, however it is encouraging that positive change was noted by those families that could engage with intervention work, suggesting that such intervention is beneficial to those that took part. In particular, it was encouraging that the number of families rating their child's sleep pattern to be a "moderate or large problem" fell from $94 \%$ of families at initial assessment, to $47 \%$ after intervention, as well as seeing Burden scores reduce from an average 4.7 out of a maximum of 6.0 , to 2.0 out of 6.0 , suggesting an important positive impact on families who had engaged.

Analysis of outcomes for those that were harder to reach, either due to non-uptake of intervention, or disengagement, was not possible due to ethical considerations of contacting families for follow-up data in the context of a clinical service rather than a research project. Future planning of research in this area will help to understand the complexities involved, and may suggest how better to serve this group of families and children in our area.

\subsection{Case Examples}

Referral: $Z$ is a 3 year old girl with Smith-Magenis Syndrome, a condition known to be associated with sleep-disturbance in $100 \%$ of cases. At time of referral she was known to have a history of obstructive sleep apnoea, and was waking multiple times through the night, with daytime behavioural challenges, and significant impact on parental wellbeing. Parents had English as an additional language, were socially isolated following recent move to the UK, and were living in one room of a shared house.

Assessment: concluded that the primary issue appeared to be one of sleep-onset association disorder, with Z requiring a bottle of milk every time she woke to successfully settle back to sleep. Repeat cardiorespiratory sleep study showed mild residual OSA and ENT referral was initiated due to parental anxiety.

Intervention: A period of psychology intervention delivered one-to-one with an interpreter helped to address feelings of parental guilt (in relation to the child's neurodisability, move to the UK, and social isolation), and through a combination of adapted Cognitive Behavioural Therapy and motivational interviewing, the family made progress with withdrawing the milk at sleep-onset time.

Impact: Following this intervention $Z$ was sleeping through the night.

Referral: $M$ is a 6-year-old girl with autism spectrum disorder with severe communication impairment (non-verbal) and severe functional impairment. She has Avoidant Restrictive Food Intake Disorder, constipation, reported allergies, eczema and hayfever. She is highly active, described as "never sitting still", and has outbursts of frustration-related aggressive behaviour. She was referred to the Sleep Clinic for difficulties with both sleep initiation (taking hours to fall asleep) and sleep maintenance (often awake for 1-2 hours during the night). She lives with both parents and her brother, sharing a bedroom with her mother. 
Assessment: Following initial assessment, referrals were made to the CAMHS Neurodevelopmental Service (behavioural intervention ongoing), Allergy Clinic (formal assessment identified multiple food and environmental allergies), GP for eczema review, and blood tests were requested.

Intervention: $M$ was found to be iron deficient, with a ferritin of $13 \mathrm{mcg} / \mathrm{L}$, and due to symptoms of restlessness in sleep (legs "jumping" at night), a course of iron medication was recommended. A trial of melatonin medication (Circadin 2mg) was suggested, alongside behavioural recommendations including structured routines, visual schedules, increasing daytime physical activity through groups accessible through the local authority Local Offer website, and use of calming sensory toys (bubble machine).

Impact: At follow up her sleep onset had improved, but sleep maintenance remained an issue.

Parents attended the Psychoeducational Workshop, however due to complex family circumstances, including illnesses, and competing priorities (such as M's feeding issues, toileting problems, and constipation), a joint decision was made to postpone further psychology support for her sleep pattern until a more useful time in the future for the family.

\section{Discussion}

We have described our experience of setting-up, running and evaluating a community-based multi-disciplinary sleep service for children with neurodevelopmental and social vulnerabilities. The data represents a "real-time" clinic population, with significant complexity and high levels of psychosocial vulnerability contributing to barriers to intervention, plus clinician factors such as staff turnover and time pressures contributing to ability, for example, to collect outcome data. Within these constraints we believe there are important conclusions that can be drawn from the findings we have presented.

\subsection{High Demand and Need for Holistic Assessment}

There has been a high demand for a community-based sleep service for this population of children. This is in keeping with reported prevalence data of sleep difficulties in children with developmental challenges, and resultant impacts on both child and family.

Holistic assessment at initial clinic contact has received very positive feedback from families and allows development of tailored recommendations to begin to address sleep-related problems, which have often been long-standing and resistant to treatment at the point of referral. We emphasise our belief that a one-hour initial assessment is necessary to fully understand the complex interplay of factors that impact on children's sleep, and begin to develop recommendations that are achievable and support individual family circumstances.

\subsection{Role of Medical Investigation}

We have accessed sleep studies for around a quarter of patients assessed. Usually this has been cardiorespiratory sleep studies to assess for sleep-disordered breathing, with polysomnography in only $3.6 \%$ and actigraphy in $5.8 \%$ (e.g. when accurate documentation of sleep pattern through history/sleep diaries was challenging or there was a need for objective evidence of sleep pattern, e.g. children under Child Protection assessment). Having access to sleep studies has helped to allay 
anxieties around potential medical factors underlying sleep issues, increasing parental confidence for putting behavioural recommendations into place. Support from the tertiary Evelina London Children's Sleep Medicine Department has been invaluable in provision and interpretation of these investigations. We acknowledge that not all services may have direct access to sleep laboratory investigations, however as the majority of studies have been related to concerns for sleepdisordered breathing, assessment could be accessed as needed through usual established clinical channels, such as Ear, Nose and Throat, or Respiratory services, where direct access is not available.

\subsection{Need for Tailored Intervention}

Experience has taught us that each family situation must be approached with tailored recommendations; suggestions that work for one family, may seem impossible to achieve for another. Setting goals that are achievable is key to maintaining momentum for change, and small steps are often needed (e.g. "start with 5 minutes outdoor activity, build up over days and weeks"). There are differing models of "multi-disciplinary" sleep services already in existence, utilising sleep practitioners, specialist nurses, occupational therapists, or psychologists - our experience has been that families often have, or gain, a good understanding of the basic recommendations to improve sleep health, however translating this into behavioural change in everyday lives has often been the challenging part. It is in this scenario that we have found psychologically based support to be particularly helpful, with specific benefit from motivational-interviewing to help support continued working towards set goals.

We have found that support from other services is often needed - e.g. Social Care (respite), CAMHS (behavioural support), Speech and Language Therapy (communication), Occupational Therapy (e.g. battles during evening routines of teeth-brushing, washing, dressing), Dietician (restricted dietary repertoires), Education (appropriate school placements), Housing (suboptimal housing conditions). We truly believe in the value of a team approach to resolving sleep-related problems, with the family as key team-members.

Around a quarter of patients received melatonin medication as part of the overall management plan, which has always included elements of behavioural change. Our clinical practice is to discuss with parents that melatonin without concurrent behavioural intervention is unlikely to be effective in the long term, and therefore runs the risk of being labelled as another treatment that failed, for families who have often tried multiple interventions in the past. Additionally, we aim to use melatonin to help set new behavioural patterns in place, recommending a trial without melatonin around every 6 months to see whether it is still needed, and to help maintain efficacy.

\subsection{Importance of Acknowledging Barriers to Intervention}

Medical and social complexity is common in our cohort. Safeguarding concerns are frequent and housing problems common (subsequent separate analysis finding $48 \%$ of children seen bedroomshared, $28 \%$ bed-shared and $24 \%$ lived in housing conditions that the clinician felt were likely to impact on sleep and success of intervention) [personal communication]. Nearly a quarter of families seen were discharged after non-engagement with the service; within this group there was a greater proportion of complexity and psychosocial adversity, with competing priorities for families. Data related to engagement with sleep interventions for children is relatively scarce within the literature, however Elphick's study [20] of a sleep intervention in vulnerable children, documented a $30 \%$ 
withdrawal rate from the study, suggesting a not dissimilar rate to our population. Acknowledging these factors and potential barriers to change is an important part of formulating appropriate steps and recommendations for the individual child and family.

Access to additional resources may help to support this cohort, and future development of the service hopes to target this.

\subsection{Future Directions}

Following the success of the pilot years of the service, we have secured recurrent funding to continue, and are now into our $4^{\text {th }}$ year of running, and continue to receive a high number of referrals ( $>700$ referrals received to date). The model of initial hour-long assessment, formulation of recommendations, and tailored intervention works well for families who are able to engage. Families experiencing a higher level of complexity and competing demands may benefit from additional resources to help effect change, and this will be explored in future development of the service.

The model we have developed we believe could be easily replicated in other community-based services, with modifications to suit local needs. However in the absence of a specialised service we would encourage all clinicians working with children with additional needs to have basic knowledge and skills in enquiring about sleep health, and be able to give basic recommendations towards improving sleep [Boxes 1 and 2], to help optimise outcomes in terms of developmental and academic progress, health and wellbeing, and family functioning.

\section{Box 1 Healthy sleep recommendations.}

Healthy sleep can only occur if the child feels safe and secure in their bedroom environment address safeguarding concerns and other night-fears/anxieties

Have a set wake-up time, and set bed-time, kept as close as possible to the same time every day Daylight exposure helps set the brain's natural daily rhythm - walking to school where possible can achieve early daylight exposure; open curtains, sit near to a window early in the day if outside time is not possible

Have plenty of physical activity during the day, preferably outdoors

Avoid caffeine and sugar

Avoid electronic screens/devices in the evening period and overnight

Have a set wind-down routine in the half-hour before bed - involving books, audio-books, calming music, simple interactive games, and avoiding screen-based activities

Always fall asleep in same place, and where child is expected to sleep for the rest of night

For children with communication impairments, develop a visual schedule for the evening and bedtime, followed in the same way each day (for examples, see resources developed by the Autism Treatment Network [3, 4])

Avoid "daytime" activities during the night-time period - e.g. coming out to living area, eating, bathing, playing 
Box 2 Tips to help encourage independent sleep (helpful for sleep-onset association disorders, where child requires parental presence, or other set conditions, in order to fall asleep successfully).

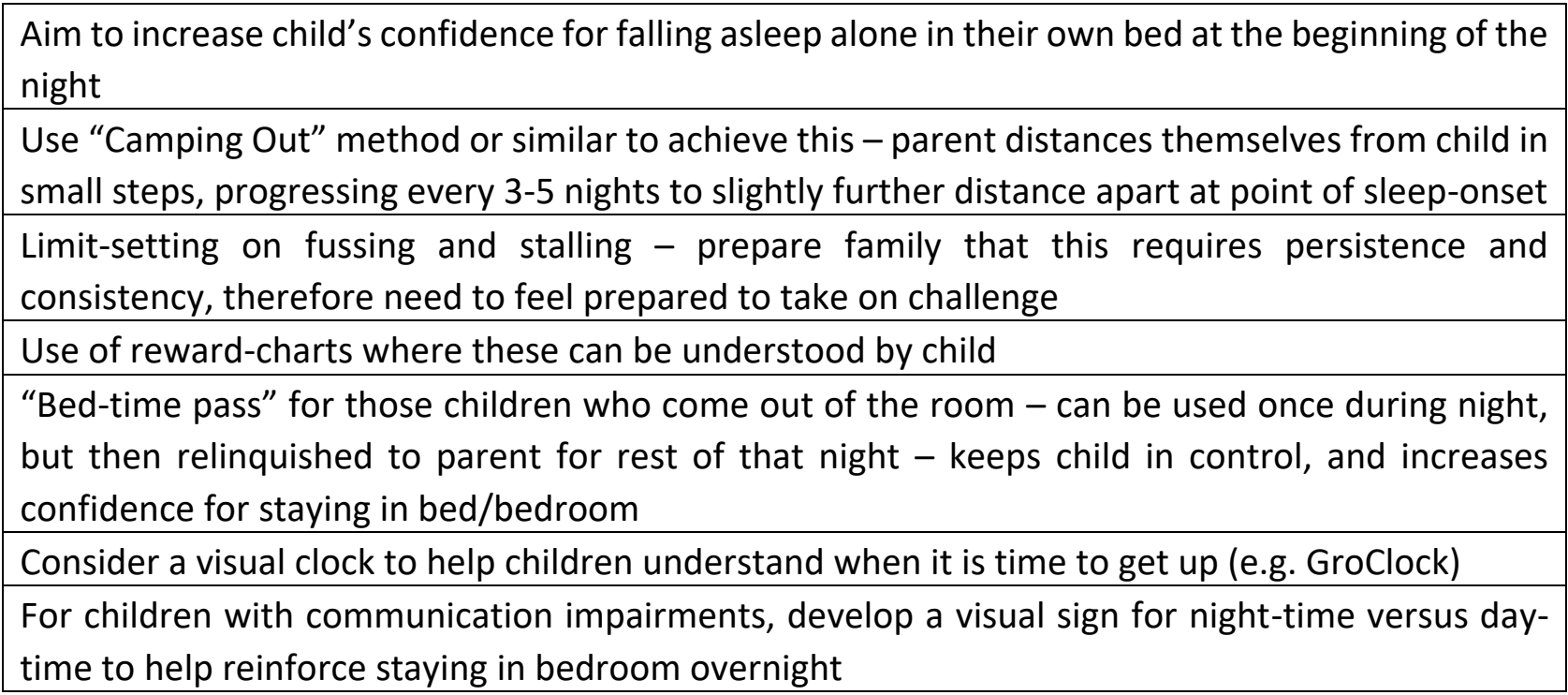

\section{Acknowledgments}

With enormous thanks to the Evelina London Children's Sleep Medicine Team for their support in the development of this service

\section{Author Contributions}

JT collated and analysed data, drafted sections of the initial manuscript, revised and edited the final version of the manuscript, and gave final approval for publication of the manuscript. SH collated and analysed data, drafted sections of the initial manuscript, revised and edited later versions of the manuscript, and gave approval for publication of the final manuscript. JVL collated and analysed data, drafted a section of the initial manuscript, and gave approval for publication of the final manuscript. BL drafted a section of the initial manuscript, critically reviewed the work for intellectual content, and gave approval for publication of the final manuscript. All authors agree to be accountable for the work and data presented.

\section{Funding}

This project received no specific grant from any funding agency in the public, commercial or notfor-profit sectors.

\section{Competing Interests}

The authors have declared that no competing interests exist. 


\section{References}

1. McDonald A, Joseph D. Paediatric neurodisability and sleep disorders: Clinical pathways and management strategies. BMJ Paediatr Open. 2019; 3: e000290.

2. Angriman M, Caravale B, Novelli L, Ferri R, Bruni O. Sleep in children with neurodevelopmental disabilities. Neuropediatrics. 2015; 46: 199-210.

3. Accardo JA. Sleep in children with neurodevelopmental disabilities: An evidence-based guide. Heidelberg, Germany: Springer; 2019.

4. Reynolds AM, Malow BA. Sleep and autism spectrum disorders. Pediatr Clin. 2011; 58: 685-698.

5. Gregory AM, Sadeh A. Sleep, emotional and behavioural difficulties in children and adolescents. Sleep Med Rev. 2012; 16: 129-136.

6. Ikeda T, Nagai T, Kato-Nishimura K, Mohri I, Taniike M. Sleep problems in physically disabled children and burden on caregivers. Brain Dev. 2012; 34: 223-229.

7. Stein MA, Mendelsohn J, Obermeyer WH, Amromin J, Benca R. Sleep and behavior problems in school-aged children. Pediatrics. 2001; 107: e60.

8. Taheri S. The link between short sleep duration and obesity: We should recommend more sleep to prevent obesity. Arch Dis Child. 2006; 91: 881-884.

9. Cortese S, Brown T, Corkum P, Gruber R, O'Brien L, Stein M, et al. Assessment and management of sleep problems in youths with attention-deficit/hyperactivity disorder. J Am Acad Child Adolesc Psychiatry. 2013; 52: 784-796.

10. Turnbull JR, Impey V, Abul-Eis H, Sharma A, Lahoti B. Sleep-related difficulties are common in children with neurodisability at both primary- and secondary-school ages. Dev Med Child Neurol. 2017; 59(S2): 46-87.

11. Turnbull JR, Farquhar M. Fifteen-minute consultation on problems in the healthy child: Sleep. Arch Dis Child Educ Pract. 2016; 101: 175-180.

12. Dosman C, Witmans M, Zwaigenbaum L. Iron's role in paediatric restless legs syndrome - a review. Paediatr Child Health. 2012; 17: 193-197.

13. Mohri I, Kato-Nishimura K, Kagitani-Shimono K, Kimura-Ohba S, Ozono K, Tachibana N, et al. Evaluation of oral iron treatment in pediatric restless legs syndrome (RLS). Sleep Med. 2012; 13: 429-432.

14. Amos LB, Grekowicz ML, Kuhn EM, Olstad JD, Collins MM, Norins NA, et al. Treatment of pediatric restless legs syndrome. Clin Pediatr. 2014; 53: 331-336

15. Herguner S, Kelesoglu FM, Tanidir C, Copur M. Ferritin and iron levels in children with autistic disorder. Eur J Pediatr. 2012; 171: 143-146.

16. Rossignol DA, Frye RE. Melatonin in autism spectrum disorders: A systematic review and metaanalysis. Dev Med Child Neurol. 2011; 53: 783-792.

17. Sung V, Hiscock $H$, Sciberras $E$, Efron D. Sleep problems in children with attentiondeficit/hyperactivity disorder: Prevalence and the effect on the child and family. Arch Pediatr Adolesc Med. 2008; 162: 336-342.

18. Gringras $P$, Gamble $C$, Jones AP, Wiggs L, Williamson PR, Sutcliffe A, et al. Melatonin for sleep problems in children with neurodevelopmental disorders: Randomised double masked placebo controlled trial. BMJ. 2012; 345: e6664. 
19. Gringras P, Nir T, Breddy J, Frydman-Marom A, Findling RL. Efficacy and safety of pediatric prolonged-release melatonin for insomnia in children with autism spectrum disorder. J Am Acad Child Adolesc Psychiatry. 2017; 56: 948-957.

20. Elphick HE, Lawson C, Ives A, Siddall S, Kingshott RN, Reynolds J, et al. Pilot study of an integrated model of sleep support for children: A before and after evaluation. BMJ Paediatr Open. 2019; 3: e000551.

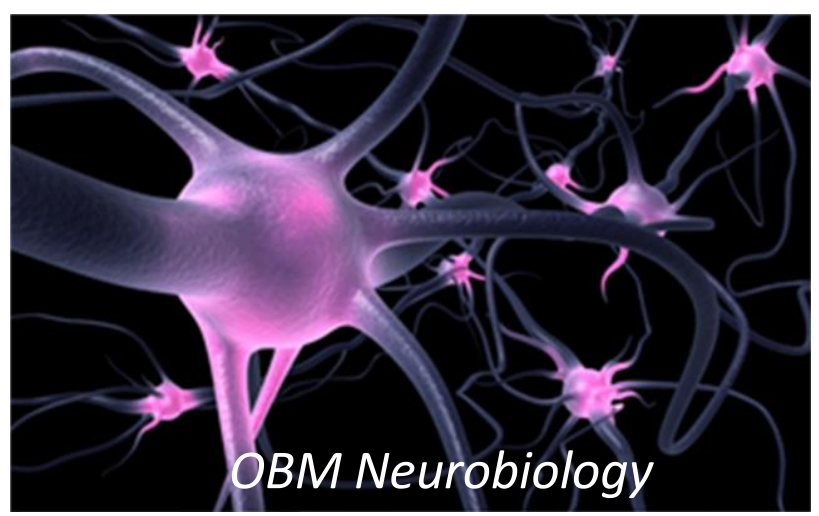

Enjoy OBM Neurobiology by:

1. Submitting a manuscript

2. Joining volunteer reviewer bank

3. Joining Editorial Board

4. Guest editing a special issue

For more details, please visit:

http://www.lidsen.com/journals/neurobiology 